\title{
Design of Cellulosic Ethanol Supply Chains with Regional Depots
}

\author{
Rex T. L. Ng1,2, Christos T. Maravelias, ${ }^{*}, 1,2$ \\ ${ }^{1}$ Department of Chemical and Biological Engineering and ${ }^{2}$ DOE Great Lakes Bioenergy Research \\ Center, University of Wisconsin-Madison, 1415 Engineering Drive, Madison, WI 53706, USA
}

\section{SUPPORTING INFORMATION}

\section{Big-M reformulation in Section 4. Remarks.}

We first calculate lower and upper bounds, $x^{\mathrm{L}} / y^{\mathrm{L}}$ and $x^{\mathrm{U}} / y^{\mathrm{U}}$, based on maximum and minimum values of the Cartesian coordinates of farms and biorefineries. The Cartesian coordinates of depots are then bounded as follows:

$x^{\mathrm{L}} \leq x_{k}^{*} \leq x^{\mathrm{U}}$

$y^{\mathrm{L}} \leq y_{k}^{*} \leq y^{\mathrm{U}}$

Equations (55) - (57) can be replaced by the following equations:

$\Delta x_{j, k} \leq\left(\Delta x_{j, l}+\epsilon\right) Z_{j, k}, \quad j, k, l$

$\Delta y_{j, k} \leq\left(\Delta y_{j, l}+\epsilon\right) Z_{j, k}, \quad j, k, l$

$D_{j, k} \leq \tau_{j, l} Z_{j, k}, \quad j, k, l$

where $\epsilon=5 \mathrm{~km}$. Note that the minimum value of $\Delta x_{j, l}$ will be essentially used as a big-M value when more than one biorefineries are considered.

The distance between the depot and the biorefinery must be less than the distance between the farthest farm and the biorefinery, which are given:

$$
\begin{aligned}
& \Delta x_{k, l} \leq \max \left\{\left|x^{\mathrm{U}}-x_{l}\right|,\left|x_{l}-x^{\mathrm{L}}\right|\right\} Z_{k, l}, \quad k, l \\
& \Delta y_{k, l} \leq \max \left\{\left|y^{\mathrm{U}}-y_{l}\right|,\left|y_{l}-y^{\mathrm{L}}\right|\right\} Z_{k, l}, \quad k, l \\
& D_{k, l} \leq\left(\max \left\{\left|x^{\mathrm{U}}-x_{l}\right|,\left|x_{l}-x^{\mathrm{L}}\right|\right\}+\max \left\{\left|y^{\mathrm{U}}-y_{l}\right|,\left|y_{l}-y^{\mathrm{L}}\right|\right\}\right) Z_{k, l}, \quad k, l
\end{aligned}
$$




\section{Data for case study in Section 5. Application.}

Table S1 gives the coordinates of farms and biorefineries, as extracted from Google Maps ${ }^{1}$. Biomass availability ${ }^{2-4}$ for each time period is tabulated in Table S2. The biomass acquisition cost and $\mathrm{CO}_{2}$ emissions during biomass collection are summarized in Table S3. Note that the biomass acquisition cost includes labor, fuel use, equipment, nutrient replacement and handling costs at farms $5,6 . \mathrm{CO}_{2}$ emissions during biomass collection includes shredding, baling and stacking7,8. Table S4 summarizes the conversion rates of all technologies which are obtained from previous works ${ }^{9-12}$. The production cost, capital cost and $\mathrm{CO}_{2}$ emissions for each technology are given in Table S5 ${ }^{9-14}$. Production cost is directly proportional to the consumption level of input materials. Capital cost of each technology is calculated based on n-th plant cost analysis and linearized it with the piecewise linearization approximation presented in Appendix A. Finally, the traveling cost and $\mathrm{CO}_{2}$ emissions due to transportation are given in Table S6 ${ }^{15}$. Note that all costs are indexed to 2014 dollars and calculated based on dry mass basis. 
Table S1: Latitude and longitude of farms and biorefineries ${ }^{1}$

\begin{tabular}{|c|c|c|c|}
\hline Node & County & Latitude & Longitude \\
\hline 0 & Origin Point & 42.505721 & -89.837117 \\
\hline \multicolumn{4}{|l|}{ Farm } \\
\hline $\mathrm{F} 1$ & Dane & 43.182533 & -89.219957 \\
\hline F2 & Dane & 43.182533 & -89.632322 \\
\hline F3 & Dane & 42.960038 & -89.219957 \\
\hline $\mathrm{F} 4$ & Dane & 42.960038 & -89.632322 \\
\hline F5 & Dodge & 43.523213 & -88.552815 \\
\hline F6 & Dodge & 43.523213 & -88.856985 \\
\hline F7 & Dodge & 43.306746 & -88.552815 \\
\hline F8 & Dodge & 43.306746 & -88.856985 \\
\hline F9 & Rock & 42.580298 & -88.925036 \\
\hline F10 & Rock & 42.580298 & -89.221768 \\
\hline F11 & Rock & 42.756749 & -88.925036 \\
\hline F12 & Rock & 42.756749 & -89.221768 \\
\hline F13 & Columbia & 43.553870 & -89.188120 \\
\hline F14 & Columbia & 43.553870 & -89.543115 \\
\hline F15 & Columbia & 43.374457 & -89.188120 \\
\hline F16 & Columbia & 43.374457 & -89.543115 \\
\hline F17 & Green & 42.769265 & -89.486714 \\
\hline F18 & Green & 42.769265 & -89.720316 \\
\hline F19 & Green & 42.593569 & -89.486714 \\
\hline F20 & Green & 42.593569 & -89.720316 \\
\hline F21 & Jefferson & 43.109021 & -88.659310 \\
\hline F22 & Jefferson & 43.109021 & -88.892769 \\
\hline F23 & Jefferson & 42.932062 & -88.659310 \\
\hline F24 & Jefferson & 42.932062 & -88.892769 \\
\hline \multicolumn{4}{|c|}{ Biorefinery } \\
\hline $\mathrm{BA}$ & Dane & 42.902712 & -89.434257 \\
\hline BB & Columbia & 43.319661 & -89.042714 \\
\hline
\end{tabular}


Table S2: Biomass availability for each time period ${ }^{2-4}$

(a) Corn stover

\begin{tabular}{ccccc}
\hline \multirow{4}{*}{ Node } & \multicolumn{4}{c}{ Period } \\
\cline { 2 - 5 } & Spring & Summer & Fall & Winter \\
\hline F1 & 0 & 24,220 & 10,380 & 0 \\
F2 & 0 & 24,220 & 10,380 & 0 \\
F3 & 0 & 24,220 & 10,380 & 0 \\
F4 & 0 & 24,220 & 10,380 & 0 \\
F5 & 0 & 23,468 & 10,058 & 0 \\
F6 & 0 & 23,468 & 10,058 & 0 \\
F7 & 0 & 23,468 & 10,058 & 0 \\
F8 & 0 & 23,468 & 10,058 & 0 \\
F9 & 0 & 20,808 & 8,918 & 0 \\
F10 & 0 & 20,808 & 8,918 & 0 \\
F11 & 0 & 20,808 & 8,918 & 0 \\
F12 & 0 & 20,808 & 8,918 & 0 \\
F13 & 0 & 19,513 & 8,363 & 0 \\
F14 & 0 & 19,513 & 8,363 & 0 \\
F15 & 0 & 19,513 & 8,363 & 0 \\
F16 & 0 & 19,513 & 8,363 & 0 \\
F17 & 0 & 8,416 & 3,607 & 0 \\
F18 & 0 & 8,416 & 3,607 & 0 \\
F19 & 0 & 8,416 & 3,607 & 0 \\
F20 & 0 & 8,416 & 3,607 & 0 \\
F21 & 0 & 10,974 & 4,703 & 0 \\
F22 & 0 & 10,974 & 4,703 & 0 \\
F23 & 0 & 10,974 & 4,703 & 0 \\
F24 & 0 & 10,974 & 4,703 & 0 \\
\hline & & & &
\end{tabular}

(b) Switchgrass

\begin{tabular}{ccccc}
\hline \multirow{2}{*}{ Node } & \multicolumn{4}{c}{ Period } \\
\cline { 2 - 5 } & Spring & Summer & Fall & Winter \\
\hline F1 & 0 & 7,712 & 3,305 & 0 \\
F2 & 0 & 7,712 & 3,305 & 0 \\
F3 & 0 & 7,712 & 3,305 & 0 \\
F4 & 0 & 7,712 & 3,305 & 0 \\
F5 & 0 & 2,927 & 1,254 & 0 \\
F6 & 0 & 2,927 & 1,254 & 0 \\
F7 & 0 & 2,927 & 1,254 & 0 \\
F8 & 0 & 2,927 & 1,254 & 0 \\
F9 & 0 & 5,142 & 2,204 & 0 \\
F10 & 0 & 5,142 & 2,204 & 0 \\
F11 & 0 & 5,142 & 2,204 & 0 \\
F12 & 0 & 5,142 & 2,204 & 0 \\
F13 & 0 & 2,527 & 1,083 & 0 \\
F14 & 0 & 2,527 & 1,083 & 0 \\
F15 & 0 & 2,527 & 1,083 & 0 \\
F16 & 0 & 2,527 & 1,083 & 0 \\
F17 & 0 & 5,020 & 2,151 & 0 \\
F18 & 0 & 5,020 & 2,151 & 0 \\
F19 & 0 & 5,020 & 2,151 & 0 \\
F20 & 0 & 5,020 & 2,151 & 0 \\
F21 & 0 & 1,595 & 683 & 0 \\
F22 & 0 & 1,595 & 683 & 0 \\
F23 & 0 & 1,595 & 683 & 0 \\
F24 & 0 & 1,595 & 683 & 0 \\
\hline & & & &
\end{tabular}

Table S3: Cost and $\mathrm{CO}_{2}$ emissions during biomass collection at farms ${ }^{5-8}$

\begin{tabular}{ccc}
\hline Compound & $\begin{array}{c}\text { Total Cost } \\
(\$ / \text { dry } t)\end{array}$ & $\begin{array}{c}\mathrm{CO}_{2} \text { Emission } \\
\left(\mathrm{kg} \mathrm{CO}_{2} \mathrm{eq} / \mathrm{dry} \mathrm{t}\right)\end{array}$ \\
\hline $\mathrm{CS}$ & 33.9 & 27.0 \\
$\mathrm{SG}$ & 55.4 & 16.0 \\
\hline
\end{tabular}


Table S4: Conversion for each technology ${ }^{9-12}$

\begin{tabular}{|c|c|c|c|}
\hline \multicolumn{2}{|c|}{ Compound } & \multirow{2}{*}{ Technology } & \multirow{2}{*}{$\begin{array}{c}\text { Conversion } \\
\text { (t output/t input } \\
\text { or L output/t input*) }\end{array}$} \\
\hline Input & Output & & \\
\hline $\mathrm{CS}$ & CS-AP & AFEX-DD & 0.99 \\
\hline SG & SG-AP & AFEX-DD & 0.99 \\
\hline CS & CS-P & AFEX & 0.99 \\
\hline SG & SG-P & AFEX & 0.99 \\
\hline CS & CS-A & AFEX & 0.99 \\
\hline SG & SG-A & AFEX & 0.99 \\
\hline CS-AP/CS-P/CS-A & Ethanol & SSCF & $280 / 280 / 278^{*}$ \\
\hline SG-AP/SG-P/SG-A & Ethanol & SSCF & $283 / 283 / 281^{*}$ \\
\hline CS-AP/CS-P/CS-A & Ethanol & SHCF & $280 / 280 / 278^{*}$ \\
\hline SG-AP/SG-P/SG-A & Ethanol & SHCF & $283 / 283 / 281^{*}$ \\
\hline
\end{tabular}

Table S5: Production cost, capacity cost and $\mathrm{CO}_{2}$ emission for each technology ${ }^{9-14}$

\begin{tabular}{ccccc}
\hline Technology & $\begin{array}{c}\text { Unit } \\
\text { Production } \\
\text { Cost } \\
(\$ / \text { dry } \mathrm{t})\end{array}$ & $\begin{array}{c}\text { Capital } \\
\text { Cost } \\
\text { Reference } \\
\left(10^{6} \$\right)\end{array}$ & $\begin{array}{c}\text { Capital Cost } \\
\text { Reference } \\
\text { Capacity } \\
\text { (dry } / \mathrm{d})\end{array}$ & $\mathrm{CO}_{2}$ Emission \\
$\left(\mathrm{kg} \mathrm{CO}_{2} \mathrm{eq} / \mathrm{dry} \mathrm{t}\right)$
\end{tabular}

Table S6: Cost and $\mathrm{CO}_{2}$ emission due to transportation ${ }^{15}$

\begin{tabular}{cccccc}
\hline & \multicolumn{2}{c}{ Cost } & \multicolumn{2}{c}{$\mathrm{CO}_{2}$ Emission } \\
\hline \multirow{2}{*}{ Arc } & \multirow{2}{*}{ Compound } & $\begin{array}{c}\text { Constant } \\
(\$ / \text { dry t })\end{array}$ & $\begin{array}{c}\text { Variable } \\
(\$ / \text { dry t-km })\end{array}$ & $\begin{array}{c}\text { Constant } \\
\left(\mathrm{kg} \mathrm{CO} \text { Cq}_{2} / \text { dry t }\right)\end{array}$ & $\begin{array}{c}\text { Variable } \\
\left(\mathrm{kg} \mathrm{CO}_{2} \mathrm{eq} / \text { dry t-km }\right)\end{array}$ \\
\hline$j \rightarrow k / j \rightarrow l$ & CS/SG & 10.7 & 0.19 & 115.9 & 1.2 \\
\multirow{2}{*}{$k \rightarrow l$} & CS-P/SG-P & 4.5 & 0.06 & 62.7 & 0.7 \\
& CS-AP/SG-AP & 3.6 & 0.06 & 28.2 & 0.7 \\
\hline
\end{tabular}




\section{References}

(1) Google Inc. Google Maps https://www.google.com/maps (accessed Aug 31, 2015).

(2) USDA Census Of Agriculture. 2012 Census Publications http://www.agcensus.usda.gov/Publications/2012/ (accessed Nov 12, 2015).

(3) Energy Biosciences Institute. Biofuel Ecophysiological Traits and Yields Database https://www.betydb.org/ (accessed Nov 12, 2015).

(4) Laboratory National Renewable Energy. BioFuels Atlas https://maps.nrel.gov/biofuels-atlas (accessed Jul 31, 2015).

(5) Brechbill, S.; Tyner, W. E. The Economics of Renewable Energy: Corn Stover and Switchgrass https://www.extension.purdue.edu/extmedia/EC/RE-3-W.pdf (accessed Jul 6, 2015).

(6) Sokhansanj, S.; Mani, S.; Turhollow, A.; Kumar, A.; Bransby, D.; Lynd, L.; Laser, M. Large-Scale Production, Harvest and Logistics of Switchgrass (Panicum Virgatum L.) - Current Technology and Envisioning a Mature Technology. Biofuels, Bioprod. Biorefining 2009, 3 (2), 124.

(7) Sokhansanj, S.; Kumar, A.; Turhollow, A. Development and Implementation of Integrated Biomass Supply Analysis and Logistics Model (IBSAL). Biomass and Bioenergy 2006, 30 (10), 838.

(8) Kumar, A.; Sokhansanj, S. Switchgrass (Panicum Vigratum, L.) Delivery to a Biorefinery Using Integrated Biomass Supply Analysis and Logistics (IBSAL) Model. Bioresour. Technol. 2007, 98 (5), 1033.

(9) Kim, S.; Dale, B. E. Comparing Alternative Cellulosic Biomass Biorefining Systems: Centralized versus Distributed Processing Systems. Biomass and Bioenergy 2015, 74, 135.

(10) Humbird, D.; Davis, R.; Tao, L.; Kinchin, C.; Hsu, D.; Aden, A.; Schoen, P.; Lukas, J.; Olthof, B.; Worley, M. Process Design and Economics for Biochemical Conversion of Lignocellulosic Biomass to Ethanol: Dilute-Acid Pretreatment and Enzymatic Hydrolysis of Corn Stover; National Renewable Energy Laboratory (NREL), Golden, CO, 2011.

(11) Aden, A.; Ruth, M.; Ibsen, K.; Jechura, J.; Neeves, K.; Sheehan, J.; Wallace, B.; Montague, L.; Slayton, A.; Lukas, J. Lignocellulosic Biomass to Ethanol Process Design and Economics Utilizing Co-Current Dilute Acid Prehydrolysis and Enzymatic Hydrolysis for Corn Stover; National Renewable Energy Laboratory (NREL), Golden, CO, 2002.

(12) Bals, B.; Wedding, C.; Balan, V.; Sendich, E.; Dale, B. Evaluating the Impact of Ammonia Fiber Expansion (AFEX) Pretreatment Conditions on the Cost of Ethanol Production. Bioresour. Technol. 2011, 102 (2), 1277.

(13) Lamers, P.; Roni, M. S.; Tumuluru, J. S.; Jacobson, J. J.; Cafferty, K.; Hansen, J. K.; Kenney, K.; Teymouri, F.; Bals, B. Techno-Economic Analysis of Decentralized Biomass Processing Depots. Bioresour. Technol. 2015, 194, 205.

(14) Eranki, P. L.; Dale, B. E. Comparative Life Cycle Assessment of Centralized and Distributed Biomass Processing Systems Combined with Mixed Feedstock Landscapes. GCB Bioenergy 2011, 3 (6), 427.

(15) Ileleji, K. E.; Sokhansanj, S.; Cundiff, J. S. Farm-Gate to Plant-Gate Delivery of Lignocellulosic Feedstocks from Plant Biomass for Biofuel Production. In Biofuels from Agricultural Wastes and Byproducts; Wiley-Blackwell, 2010; pp 117-159. 